\title{
El cuarto árbitro: la escala de grises en la función del secretario administrativo
}

\author{
Orlando F. Cabrera Colorado* \\ Recibido/Received: 25/09/2017 \\ Aceptado/Accepted: 20/10/2017
}

SUMARIO: 1. La problemática. 2. El mandato arbitral y la idoneidad de un secretario arbitral. 3. Principios rectores. 3.1 Imparcialidad e independencia del secretario arbitral. 3.2 Actividades permitidas para el secretario administrativo. 3.3 Deliberaciones. 3.4 Actividades prohibidas para el secretario administrativo. 3.5 La remuneración del secretario administrativo. 4. Conclusiones.

Palabras Clave: secretario administrativo, asistente del tribunal arbitral, funciones.

KeYWORDs: administrative secretary, assistant to the arbitral tribunal, roles.

RESUMEN: La función del secretario administrativo o asistente de un tribunal arbitral transita por una escala de grises que genera preocupaciones en cuanto a la legitimidad del procedimiento arbitral. Si bien existen algunos instrumentos de soft law que brindan certidumbre a las partes en cuanto a las funciones que el tribunal arbitral puede delegar al secretario ad-

* Foreign Law Clerk en Hogan Lovells US LLP. Licenciado en Derecho magna cum laude por la Universidad de las Américas Puebla (México), Maestro en Derecho por New York University School of Law. Correo electrónico: orlando.cabrera@hoganlovells.com

O. F. CABRERa COLORADO, "El cuarto árbitro: la escala de grises en la función del secretario administrativo”, Revista Ecuatoriana de Arbitraje, No. 9, 2017. 
ministrativo, resultan insuficientes. Algunos reglamentos de arbitraje dejan a la discrecionalidad del tribunal arbitral el rol de este asistente. No es de sorprenderse que esta discrecionalidad genere una problemática pues la falta de estándares crea diferentes percepciones. Se han intentado anular laudos arbitrales argumentando una extralimitación de funciones por parte del secretario arbitral. Para describir la escala de grises, se comenta la problemática, la naturaleza del mandato arbitral, las actividades que idealmente tienen permitidas los secretarios y aquéllas que tienen prohibidas; así como la percepción que la comunidad arbitral tiene respecto de estos asistentes.

Abstract: The role of the administrative secretary or assistant to the arbitral tribunal navigates through a grayscale generating concerns in regards to the arbitration's legitimacy. Even though some instruments of soft law provide certitude to the parties concerning the tasks that the arbitral tribunal may delegate to the administrative secretary, these remain insufficient. Some arbitration rules leave to the discretion of the arbitral tribunal the task to determine the role of the administrative secretary. Not surprisingly, this discretion causes problems, the lack of standards generates diverse perceptions. Parties have tried to set aside arbitral awards alleging that the administrative secretary exceeded its functions. To describe the grayscale, we explore the problem, the nature of the arbitral mandate, the activities ideally permitted to the secretary and those impermissible ones, as well as the perception of the arbitral community regarding these assistants.

\section{La Problemática}

Frecuentemente, surgen preguntas en el arbitraje internacional respecto al rol que desempeña el secretario del tribunal arbitral para el cual los reglamentos de arbitraje u otros instrumentos 
relevantes de soft law ${ }^{1}$ no contienen respuestas claras. Incluso, se ha dicho, que en el arbitraje internacional, el rol del secretario administrativo se presenta como una "enorme área gris" objeto de múltiples abusos².

Bajo esta área gris, Sonatrach de Algeria demandó la nulidad de un laudo ICC que le condenaba a pagar USD \$536'000,000 a favor de Statoil. El segundo argumento, con el cual Sonatrach intentó la nulidad consistió en que el secretario participó indebidamente en las deliberaciones del tribunal. Sin embargo, la Corte Superior de Inglaterra y Gales desechó la solicitud de Sonatrach ${ }^{3}$.

El ejemplo más representativo de esta zona de grises se presentó con los laudos arbitrales emitidos en tres casos que inició OAO Yukos Oil Company en contra de Rusia. Derivado de estos arbitrajes, el tribunal arbitral condenó a Rusia a pagar más de 50 mil millones de dólares. Rusia buscó la nulidad ante un Juzgado de Distrito en La Haya, Países Bajos, alegando, entre otros argumentos, que los árbitros no desempeñaron personalmente su mandato porque el asistente del tribunal jugó un papel protagónico en el análisis de pruebas y argumentos, en las deliberaciones del tribunal y en la redacción del laudo 4 . Para Rusia, los árbitros delegaron responsabilidades sustantivas al asistente del Tribunal; por tanto, violaron el mandato de realizar sus responsabilidades personalmente. En otras palabras, el asistente actuó como el cuarto árbitro.

En su momento, YvEs FORTIER, el Presidente del Tribunal Arbitral, informó a las partes que designó a Martin Valasek para "asistirme en la conducción del caso". FORTIER refirió "porque,

1. El término soft law se refiere a aquellos estándares, códigos, principios, lineamientos y tratados que no tienen carácter vinculante, en contraste con las normas de derecho obligatorio impuestas por leyes arbitrales y tratados internacionales, así como el marco procesal elegido por las partes a través de un reglamento de arbitraje. W.W. PARK, "Chapter 7: The Procedural Soft Law of International Arbitration: Non-Governmental Instruments”, en J. D. M. LEW y L. A. Mistelis, Pervasive Problems in International Arbitration, Kluwer Law International, 2006, pp.141-154.

2. M. Polkinghorne y C. B. Rosenberg, "The Role of the Tribunal Secretary in International Arbitration: A Call for a Uniform Standard", Dispute Resolution International, Vol. 8 (2), 2014, p. 107.

3. England and Wales High Court, Sonatrach v. Statoil, 02/04/2014, párr. 46-50.

4. D. Galagan, "The Challenge of the Yukos Award: an Award Written by Someone Else - a Violation of the Tribunal's Mandate?", Kluwer Arbitration Blog, <goo.gl/KkT4J2>, (19/09/2017). 
como todos nosotros, yo viajo mucho, si en algún momento no estoy localizable, ustedes siempre pueden contactarlo" ${ }^{\prime \prime}$. El nombramiento fue hecho sin previa consulta de las partes, pero cuando se reveló, las partes no lo objetaron ${ }^{6}$.

Rusia buscó anular el laudo arguyendo que la delegación de responsabilidades arbitrales al asistente se manifiesta en la desproporcionada facturación de horas hecha por Valasek misma que ascendió la cantidad de EUR 970,562 mientras que los honorarios de los árbitros -Charles Poncet, Stepahn M Schewebel e Yves Fortier- fueron por EUR 1'513,880, EUR 2'011,092 y EUR 1'732,937 respectivamente. Aplicando una tarifa por hora del asistente de EUR 250-325 y de cada árbitro de EUR 750-850, Rusia argumentó que el asistente empleó más horas que cualquiera de los árbitros especialmente en la fase de méritos del caso. En total, Valasek trabajó 3,006 horas en los arbitrajes de Yukos, tan solo 381 horas en las audiencias de jurisdicción y admisibilidad, así como unas 2,625 horas tanto en la fase de méritos como en la redacción del laudo. Esto es un 40 a 70\% más que el número de horas empleadas por cualquiera de los árbitros ${ }^{7}$.

Rusia consideró que el tiempo empleado por Valasek no se explica en su rol administrativo o logístico porque dos miembros de la Corte Permanente de Arbitraje de la Haya emplearon más de 5,200 horas asistiendo también en estos arbitrajes. Rusia solicitó los detalles del trabajo desempeñado por el asistente, pero esta solicitud fue rechazada con el argumento que esto pondría en peligro "la confidencialidad de las deliberaciones del Tribunal". Para Rusia, esto significó una admisión por el Tribunal que Valasek participó en las deliberaciones del caso $^{8}$.

El exceso en el cobro de honorarios y otras quejas, no son del todo nuevas. El anterior Secretario General de la ICC, ERIC SCHWARTZ, comentó en 1996 que la ICC había recibido quejas al

\footnotetext{
5. L.W. Newman y D. Zaslowsky, "The Yukos Case: More on the Fourth Arbitrator", International Dispute Resolution, New York Law Journal, (28/05/2015), <goo.gl/kyF3as>, (18/09/2017), p. 2.

6. Ibídem.

7. D. Galagan, N. 4.

8. Ibídem.
} 
respecto. Las partes reclamaron que en diversas ocasiones sintieron que no podían objetar el nombramiento del secretario administrativo. Además, las cantidades por honorarios del secretario administrativo, en ocasiones, fueron muy altas, incluso iguales a los honorarios de los árbitros ${ }^{9}$. Del mismo modo, ScHWARTZ reportó que al menos en un caso, la Corte de la ICC requirió al Tribunal que reemplazara al secretario porque actuaba como un árbitro ${ }^{10}$.

Esta problemática respecto a las funciones del secretario o asistente de un tribunal arbitral se ve acentuada por otras dudas relativas a las facultades del tribunal para imponerlo. Aunque en el año de 1990 la Corte de Apelaciones de París resolvió que un tribunal arbitral tiene la "posibilidad de designar un secretario"11 e incluso algunos han señalado que es una práctica arbitral en el arbitraje de inversión designar asistentes ${ }^{12}$, han existido pocos intentos de considerar completamente la fuente, naturaleza y alcance de la función del secretario arbitral, así como su relación con el tribunal arbitral. Este es el objeto de análisis del presente trabajo.

Los puntos antes señalados son oportunos en virtud de la prevaleciente incertidumbre concerniente a la intervención del secretario administrativo en el arbitraje. Incertidumbre misma que ha cuestionado la legitimidad del arbitraje. También este artículo resulta oportuno en vista de la creciente aparición de múltiples instrumentos, principalmente de soft law, que reflejan una regulación disonante al grado que es posible sostener que no existe uniformidad en la regulación de las funciones de los secretarios administrativos. Esto pone de manifiesto la existencia de un área gris en el arbitraje internacional.

9. E. A. Schawrtz, "On the subject of 'Administrative Secretaries': A Reply by Mr. Eric Schwartz, Secretary General of the ICC", ASA Bulletin, Vol. 14 (1) 1996, pp. 32-34.

10. E. A. SCHAWRTz, "The Rights and Duties of ICC Arbitrators", en ICC International Court of Arbitration Bulletin Special Supplement: The Statute of the Arbitrator, 1995, p. 86.

11. Corte de Apelaciones de París, Compagnie Honeywell Bull S.A. v. Compatacion Bull de Venezuela C.A. $(20 / 06 / 1990)$.

12. Véase, D. Galagan N. 4; UNCITRAL, 2016 UNCITRAL Notes on Organizing Arbitral Proceedings, párr. 35. "Some arbitral institutions routinely assign secretaries to cases administered by them. Where this is not the case, some arbitrators frequently engage secretaries, particularly in large or complex cases $[\ldots]$...' 
Encontramos una pluralidad de instrumentos tales como las Notas de la CNUDMI de 2016 sobre la organización del proceso arbitral, Note to Parties and Arbitral Tribunals on the Conduct of the Arbitration under the ICC Rules of Arbitration (2016), SIAC Practice Note on the Appointment of Administrative Secretaries (2015), HKIAC Guidelines on the Use of Secretary of the Arbitral Tribunal (2014), SCC's Arbitrator's Guidelines (2014), Finland Arbitration Institute's Note on the Use of a Secretary (2013); ICC Note on Appointment, Duties and Remuneration of Administrative Secretaries (2012), JAMS Guidelines for Use of Clerks and Tribunal Secretaries in Arbitrations (2012), Reglamento Administrativo y Financiero del CIADI (2006), AAA - Code of Ethics for Arbitrators in Commercial Disputes y LCIA's position on the appointment of Secretaries to Tribunals. No se puede pasar por alto que Young ICCA, sin ser una institución arbitral, ha hecho un esfuerzo importante al publicar su Guide on Arbitral Secretaries (2014).

La reciente proliferación de lineamientos de soft law sugiere que los usuarios del arbitraje buscan que sea predecible. Una mayor regulación de soft law de cierta forma constriñe la flexibilidad, concepto que cambia de color dependiendo el contexto, cosa que no debe sorprender para una palabra que se define como la habilidad de adaptarse en respuesta a nuevas situaciones. Pero al mismo tiempo, la regulación de soft law forja un sentido de igualdad de trato, promoviendo la percepción que el procedimiento es "normal" y conforme al principio de estado de derecho. Es deseable que casos similares sean tratados de forma similar. El potencial beneficio del soft law procesal radica en que mejora la percepción de justica en la resolución de controversias. Esto ayuda a mantener el equilibrio entre lo que es justo y eficiente. Toda vez que el arbitraje implica respeto al debido proceso -a la obligación de ser oído- el debido proceso se encuentra en el centro de lo que los litigantes buscan en el arbitraje ${ }^{13}$.

Mientras no existan expectativas acerca de la forma normal o regular de hacer las cosas, los litigantes carecen de supuestos de lo que es justo. La ausencia de estándares definidos con anti13. W.W. PARK, N. 1, párr. 7-12; 7-17; 7-28; 7-29. 
cipación puede generar sentimientos de falta de igualdad. La existencia de dos diferentes líneas de medida de cualquier decisión ex post que haga el árbitro se va a desviar de una de las impresiones de integridad procesal que tengan las partes ${ }^{14}$. Los lineamientos de soft law han evolucionado para mitigar algunos de los riesgos de la discreción arbitral.

Este artículo inicia señalando el mandato arbitral y la idoneidad de un secretario arbitral para la eficiente conducción de un arbitraje. Posteriormente, nos referimos a la percepción común de algunas partes hacia la existencia de secretarios y su intervención en el arbitraje. Acto seguido, se expondrán los principios comunes entre estos instrumentos de soft law. Finalmente, pasaremos a señalar la problemática existente en esta escala de grises que transita la función del secretario arbitral.

\section{El MANDATO ARBitRAL Y LA IDONEIDAD DE UN SE- CRETARIO ARBITRAL}

La misión del árbitro tiene una naturaleza intuitu personae. La elección de una parte respecto de su árbitro es personal, y así también resulta el mandato del árbitro elegido. Al aceptar el nombramiento, el árbitro necesariamente acepta la obligación de no delegar su mandato ${ }^{15}$, cuyo núcleo consistente en resolver la controversia que se le plantea. PARTASIDES hace notar que esta obligación personal está implícita en el artículo 2.3 de las Reglas de la IBA sobre Ética para Árbitros Internacionales. Este artículo señala "sólo si él está dispuesto a dar al arbitraje el tiempo y la atención que las partes razonablemente tienen derecho a esperar"16. Con lo cual se infiere que es el árbitro nombrado y no otra persona la que debe dedicar tanto tiempo como atención en la resolución de la disputa.

\footnotetext{
14. Ídem, párr. 7-34.

15. C. PARTASIDES, "The Fourth Arbitrator? The Role of Secretaries to Tribunals in International Arbitration”, Arbitration International, Vol. 18 (2), 2002, p. 147.

16. Ibídem.
} 
Es cierto que en diversas jurisdicciones nacionales las cortes utilizan el auxilio de asistentes, secretarios $\mathrm{u}$ otros profesionales para desempeñar la función judicial, pero Partasides hace notar algunas importantes diferencias entre el sistema judicial y el arbitral. Primero, las partes eligen al árbitro, no así al juez. La relación entre el juez y la parte no es intuitu personae. Segundo, los jueces -por lo general ${ }^{17}$ - no pueden controlar el volumen de casos que llega a sus juzgados; los árbitros por el contrario, tienen un absoluto control del número de tribunales en los cuáles deciden fungir como árbitros. El árbitro es el único responsable de no tener tiempo para dedicarle al $\operatorname{caso}^{18}$, bien puede rechazar un nombramiento si ya tiene una cantidad absorbente de trabajo ${ }^{19}$, que el impida dedicar tiempo suficiente para resolver un arbitraje más.

En una entrevista previa al nombramiento, las partes antes de elegir al árbitro -además de verificar que no exista conflicto de interés y otros temas- pueden preguntar si éste tiene tiempo ${ }^{20}$. Dependiendo de la respuesta que dé el árbitro, las partes pueden desistir de nombrar al potencial candidato en vista de la sobrecarga de trabajo que este profesional tenga. De otra forma, las partes conscientes que el potencial candidato tenga ya múltiples nombramientos ${ }^{21}$, también implícitamente aceptan el riesgo que el árbitro no tenga tiempo para hacer todas y cada una de las actividades derivadas de la función arbitral. De otra forma, las partes bien podrían optar por elegir un árbitro que tenga pocos o ningún nombramiento para que haga todas y cada una de las tareas que implica el ser árbitro. Incluso, las partes podrían llegar

17. La Corte Suprema de Estados Unidos de América es una excepción, ya que admite sólo unos 100 a 150 writs of certiorari al año, en la medida que estos tengan una trascendencia nacional, sirvan para armonizar una contradicción de criterios entre las cortes federales de circuito, y/o tenga un importante valor para sentar un precedente. US Courts, Supreme Court Procedures, <goo.gl/uJWJVk>, (20/09/2017).

18. El Comentario al Artículo 2, inciso (e) de la Guía del Chartered Institute of Arbitrators refiere: “Before deciding to accept any appointment, prospective arbitrators should determine whether they can devote the necessary time and attention to remain available throughout the proceedings and ensure expeditious and efficient conduct of the arbitration". Chartered Institute of Arbitrators, Interviews for Prospective Arbitrators, <goo.gl/aK37Cy>, (20/09/2017).

19. C. Partasides, N. 14, p. 147.

20. Chartered Institute of Arbitrators, N. 17, Art. 2.

21. ICCA, "Young ICCA Guide on Arbitral Secretaries", The ICCA Reports No. 1 <www.youngicca.org> (21/09/2017). 
a exigir, en un acta de misión u otro instrumento, que los árbitros se obliguen a no delegar la función, si es que tuvieran esta preocupación.

Ante estas circunstancias, los árbitros que tengan una carga abundante de compromisos y acepten un nombramiento más para actuar en un tribunal arbitral, bien pueden echar mano del apoyo que un secretario administrativo o asistente le puede brindar a un tribunal arbitral. Además, sumando otras variables como la multiplicidad de partes en un arbitraje, el abundante intercambio de comunicaciones, el volumen de un expediente, la complejidad de una disputa y otras tareas administrativas favorecen la idea de tener ayuda adicional para ejercer la función arbitral de forma más eficiente ${ }^{22}$.

Incluso, cabe señalar que el papel de un secretario arbitral es una escuela de aprendizaje que bien justifica la necesidad de enseñar y entrenar a las nuevas generaciones ${ }^{23}$. Pero a la vez, PARTASIDES subraya que los principiantes no sólo aprenden de la observación sino del hacer. Esta es su preocupación, que los secretarios sirvan como aprendices pero que hagan el trabajo del árbitro poniendo en riesgo la legitimidad del arbitraje ${ }^{24}$. Estas preocupaciones y la falta de claridad en diversos instrumentos de soft law llevaron a Young ICCA a tratar de entender la percepción de la comunidad arbitral a través de dos sondeos ${ }^{25}$.

En 2012, Young ICCA realizó un sondeo que arrojó que 95\% de los encuestados aprueba el uso de los secretarios arbitrales, principalmente para actividades administrativas como organizar juntas y audiencias con las partes, manejar correspondencia y pruebas, así como recordar a las partes de juntas y fechas de término. Los encuestados se vieron menos favorables respecto a la posibilidad que los secretarios arbitrales hagan investigaciones jurídicas para el tribunal arbitral, redacten órdenes procesales, re-

22. Véase, ibídem; F. Mantilla Serrano, "Uso y abuso del secretario del tribunal arbitral”, Legis, <ambitojuridico.com>, (18/09/2017).

23. C. Partasides, N. 14; F. Mantilla, N. 21.

24. C. PARTASides, N. 14, p. 149.

25. ICCA, N. 20. 
alicen comunicaciones con las partes en nombre del tribunal arbitral, comunicaciones con la institución y redacten partes del laudo, así como analicen los escritos de las partes. Los encuestados favorecieron la idea que el secretario sea un abogado joven, éste es el perfil ideal que debe tener un secretario arbitral para un $89 \%$. La mayoría favoreció la idea que sean las partes quiénes paguen los honorarios del secretario arbitral ${ }^{26}$.

Posteriormente, Young ICCA llevó a cabo otro sondeo en 2013 donde se puede entender mejor la percepción de la comunidad arbitral respecto de la función del secretario. La mayoría está a favor que el secretario arbitral esté admitido para ejercer en una jurisdicción, sin que sea necesario que cuente con experiencia profesional. Un $94 \%$ de los encuestados favoreció que el tribunal arbitral proponga el nombramiento del secretario arbitral y un $81 \%$ está de acuerdo que sea el tribunal quien lo designe. Sin embargo, casi el 80\% está de acuerdo en que sean las partes quienes consientan al nombramiento del secretario arbitral, así como la necesidad de que presenten una declaración de independencia e imparcialidad. Este sondeo coincide con el anterior, pues la mayoría favorece que el secretario arbitral organice cuestiones administrativas. Empero, un $83.5 \%$ está en contra que el secretario arbitral participe en las deliberaciones del tribunal. A diferencia del sondeo anterior, en el sondeo de 2013 la mayoría está de acuerdo en que sea el tribunal arbitral quien pague los costos del secretario arbitral $(60.5 \%)$.

Si bien existen similitudes en el sondeo, lo cierto es que los puntos de consenso entre los instrumentos de soft law resultan mínimos. A continuación, se exponen esos escasos puntos en relación con la función del secretario arbitral donde existe consenso y otros que parecen prudentes para seguir avanzando la uniformidad de esta figura encaminada a la eficiencia del arbitraje. 


\section{Principios ReCtores}

POLKINGHORNE y ROSENBERG postulan ciertos estándares que deben ser atendidos para poder definir el rol del secretario arbitral $^{27}$. Algunos de estos estándares encuentran el reconocimiento de diversas instituciones; sin embargo, otros quedan sin atención por las instituciones y prosiguen en el área gris. Bien esta área gris se puede erigir como un nicho de oportunidad. A continuación, pasamos a exponer los estándares y a enunciar los preceptos de soft law que los reconocen para determinar el estatus que guarda la regulación.

\subsection{Imparcialidad e independencia del secretario arbitral}

A fin de identificar conflictos de interés y proveer a las partes con una razonable oportunidad de objetar el nombramiento del secretario arbitral, el candidato a secretario debe firmar una declaración de imparcialidad e independencia revelando cualquier circunstancia probable que justifique dudas relacionadas a su actuar. Esta obligación de revelar permanece vigente aún durante su desempeño como secretario arbitral una vez nombrado ${ }^{28}$.

Este primer estándar se ve reflejado en diversos instrumentos. La Nota de la ICC de 2016 en el párrafo 79 refiere que los secretarios administrativos deben satisfacer los mismos requisitos de imparcialidad e independencia que los árbitros bajo el reglamento ICC. Asimismo, esta Nota de 2016 refiere que el tribunal arbitral debe presentar a las partes el currículum vitae del potencial secretario, una declaración de imparcialidad e independencia, un compromiso del secretario de actuar conforme a esta Nota y un compromiso del tribunal arbitral donde se asegurará que esta obligación del secretario administrativo se cumpla. La Nota de la ICC de 2016 incorporó la totalidad del texto que apareció en la Nota de la ICC de 2012, por lo cual en lo sucesivo sólo nos referiremos a la Nota más reciente. De esta forma, la ICC cumple con este estándar.

27. M. Polkinghorne y C. B. Rosenberg, N. 2, p. 122.

28. Ibídem. 
La Nota de la ICC de 2016 refiere en el párrafo 80 que el tribunal arbitral debe aclarar a las partes que ellas pueden objetar esta propuesta y el secretario no debe ser nombrado si las partes le han objetado. Este párrafo parece atender la queja que tuvieron las partes durante la gestión de Eric Schwartz como Secretario General de la ICC durante la década de los años 90. Ciertamente, parece razonable que un secretario administrativo no sea designado si las partes objetan su actuación en el arbitraje.

LCIA en la sección de Frequently Asked Questions refiere que los secretarios requieren la verificación del conflicto de intereses de rutina ${ }^{29}$. La Nota de SIAC de 2015 en su párrafo 4, obliga al secretario administrativo a firmar una declaración de independencia e imparcialidad y confidencialidad previo a su nombramiento. Por último, HKIAC en sus Guidelines de 2014 exigen en el numeral 2.2 que el candidato a secretario haga su declaración de disponibilidad, imparcialidad e independencia, así como revele cualquier circunstancia que pueda dar motivo a dudas de su imparcialidad e independencia. También en el punto 2.3 estos lineamientos obligan a presentar el currículum del potencial secretario. JAMS en sus Guidelines de 2012 establece que los secretarios requieren una revelación de conflictos de intereses por separado.

\subsection{Actividades permitidas para el secretario administrativo}

Las actividades permitidas para el secretario arbitral se refieren a las actividades administrativas y otras de asistencia que puede dar al tribunal, mismas que están reflejadas en las Notas de la ICC, los HKIAC Guidelines y la Nota de la CNUDMI de 2016. Por una parte, se permite que el secretario organice y administre ciertas tareas, incluyendo la transmisión de comunicaciones a nombre del tribunal arbitral, organizar y mantener los expedientes del tribunal, así como coordinar la logística y servicios secretariales.

29. LCIA, Frequently Asked Questions, <goo.gl/4WBsuQ>, (20/09/2017). 
Por otra parte, se acepta que el secretario pueda investigar cuestiones fácticas y jurídicas en el expediente siguiendo la dirección detallada del tribunal arbitral. El secretario no puede buscar cuestiones fácticas fuera del expediente. POLKINGHORNE y ROSENBERG señalan que atendiendo el volumen de documentos ofrecidos como pruebas en arbitrajes internacionales complejos, puede resultar eficiente por tiempo y costos que el secretario busque ciertos hechos y cuestiones jurídicas en el expediente en lugar que sean los árbitros quienes dediquen muchas horas buscando detalles en los documentos ${ }^{30}$.

Estos autores citan como ejemplos permisibles de actividades de investigación la preparación de la cronología de hechos y resumen de fuentes jurídicas. No obstante, hacen notar, que cualquier investigación que haga el secretario administrativo debe ser bajo la dirección detallada de un miembro del tribunal para minimizar el riesgo que el secretario omita inadvertidamente o tergiverse hechos o disposiciones jurídicas clave ${ }^{31}$.

PARTASIDES incluso sugiere que en aquellos casos donde la competencia no sea objeto de disputa, el árbitro, puede legítimamente hacer un primer borrador de aquellas partes del laudo identificando las partes y describiendo las bases de la competencia del tribunal arbitral sin sacrificar el control de la toma de decisiones. Igualmente sugiere, que en casos donde las decisiones procesales no sean controvertidas el árbitro puede responsablemente encargar al secretario un primer borrador de parte del laudo en que se describa el procedimiento. Incluso, va más allá y considera que en casos excepcionales, cuando los hechos de la controversia o su resultado sean para el tribunal suficientemente claros, no se sacrifica la toma de decisiones teniendo al secretario que haga un primer borrador de parte del laudo en el que se describe la disputa o se discuten los méritos ${ }^{32}$.

30. M. Polkinghorne y C. B. Rosenberg, N. 2, p. 122.

31. Ibídem.

32. C. Partasides, N. 14, p. 158. 
Contrario a lo anterior, el Profesor DALHUISEN sostiene que el secretario no debe preparar porción alguna del laudo. Para él, los argumentos clave y relevantes, así como la forma en que han de presentarse en el laudo final corresponde a los árbitros o al comité ad hoc. Así, a DALHUISEN le resulta inapropiado que el secretario prepare parte o toda la decisión y el razonamiento, incluso si fuera bajo las instrucciones de los árbitros ${ }^{33}$. La diferente forma de pensar que tienen PARTASIDES y DALHUISEN pueden ser motivos para elegirles o no como árbitros. Esto también dependerá del estilo de las partes que los nombren como coárbitros.

Estos dos estándares están insertos en la Nota de la ICC de 2016, numeral 83, al explicar con detalle que el secretario puede realizar actividades administrativas como transmitir documentos y comunicaciones a nombre del tribunal arbitral; organizar y mantener el expediente del tribunal arbitral así como localizar documentos; organizar audiencias y juntas; presenciar audiencias, juntas y deliberaciones, tomando el tiempo, notas o minutas; conducir investigaciones jurídicas o similares; pruebas de lecturas, revisión de citas, fechas, referencias cruzadas en tanto en órdenes procesales como laudos, así como corregir errores ortográficos, gramaticales y de cálculo.

Esta Nota en el punto 86 recuerda que la función arbitral es intuito personae, pues independientemente de las notas o memorándums que prepare el secretario, no releva al tribunal arbitral de su obligación personal de revisar el expediente y rectar cualquier decisión que le competa. Este numeral parece estar en contra del razonamiento que predica PARTASIDES, por lo cual, se ve difícil que tenga cabida su pensamiento en el arbitraje ICC.

Cabe decir que las dos Notas de la ICC están armonizadas con los HKIAC Guidelines de 2014 numerales 3.3. y 3.6. Los HKIAC Guidelines agregan a lo anterior que los secretarios pueden recolectar las facturas de los árbitros y realizar otros asuntos administrativos que no sean responsabilidad del HKIAC. Estos

33. Compañia de Aguas del Aconquija SA \& Vivendi Universal SA v. Argentine Republic, ICSID Case No. ARB/97/3, Procedimiento de Anulación, Opinión Adicional del Professor J. H. Dalhusein, 30/07/2010. 
lineamientos en el numeral 3.4 permiten otras actividades siempre que no se influencie la decisión del tribunal arbitral como hacer investigaciones jurídicas o similares, recopilar casos y doctrina, verificar citas, buscar cuestiones fácticas, preparar resúmenes de casos y las posiciones de las partes, presenciar las deliberaciones del tribunal y tomar notas, redactar borradores de cartas no sustantivas para el tribunal arbitral así como partes no sustantivas de órdenes, decisiones y laudos tales como la historia procesal y cronología de eventos.

Las Notas de la CNUDMI de 2016 en el punto 36 refieren que los secretarios pueden brindar apoyo puramente técnico como hacer reservaciones de audiencias y salas de juntas; así también coordinar y dar servicios administrativos. Igualmente señala que los tribunales pueden solicitar que los secretarios hagan investigaciones jurídicas, resúmenes de hechos, o la historia procesal del arbitraje, recopilando y haciendo resúmenes de casos, doctrina, y borradores de decisiones procesales.

LCIA es limita al decir que el secretario puede organizar papeles para el tribunal guardando las hojas de tiempos del tribunal y cosas similares ${ }^{34}$. El Reglamento Administrativo y Financiero del CIADI establece en la Regla 25 que el secretario levantará actas resumidas de las audiencias a menos que se acuerde algo distinto. En un 50\% de los casos, las partes y árbitros utilizan al secretario administrativo en los procedimientos CIADI, como un intercesor entre las partes y el tribunal arbitral para transmitir comunicaciones y temas administrativos.

\subsection{Deliberaciones}

Presenciar las deliberaciones ha sido un tema escabroso y motivo de argumentos de nulidad en el caso de Sonatrach v. Statoil que comentamos al principio de este artículo. El secretario puede presenciar deliberaciones si todos los miembros del tribunal lo consienten. Puede resultar eficiente que el secretario esté presente 34. M. Polkinghorne y C. B. Rosenberg, N. 2, pp. 126-127. 
en las deliberaciones, especialmente si el secretario va a preparar proyectos de órdenes procesales y partes del laudo no relacionadas con el fondo del asunto. Inclusive, POLKINGHORNE y RONSENBERG señalan que el tribunal arbitral necesita acceder rápidamente a los documentos del caso durante las deliberaciones, por lo que el secretario puede ayudar a facilitar esta tarea al tribunal ${ }^{35}$.

No obstante, se sugiere que la presencia del secretario sea condicionada a que todos los miembros del tribunal lo consientan como lo estipula la Regla de Arbitraje CIADI 15(2). Esta regla refiere: "(1) Las deliberaciones del Tribunal se realizarán en privado y permanecerán secretas. (2) Sólo los miembros del Tribunal tomarán parte en sus deliberaciones. Ninguna otra persona será admitida, a menos que el Tribunal decida otra cosa"36. Las deliberaciones generalmente son secretas para asegurar un intercambio libre de ideas sin obstáculos. Empero, la presencia del secretario no debe perjudicar la franqueza en la discusión de forma tal que sea un detrimento en la toma de decisión. La función del secretario debe quedar estrictamente limitada a transcribir el razonamiento del tribunal y sus decisiones. El secretario no debe participar activamente en las deliberaciones ${ }^{37}$. No existen instrumentos que soporten este nivel de detalle pero pueden resultar eficientes estas ideas.

\subsection{Actividades prohibidas para el secretario administrativo}

El secretario no deberá redactar partes relativas a la sustancia del laudo. La sustancia es el núcleo del arbitraje, la resolución misma de la controversia. Por consiguiente, la redacción de la sustancia y fondo del laudo concierne a los árbitros en virtud que la función arbitral es intuito personae. De lo contario, POLKINGHORNE y RONSENBERG hacen notar que existe un riesgo alto de que el razonamiento o la sección dispositiva de un laudo lleve consigo la perspectiva del secretario influenciando indebidamente el juicio

35. Ibídem.

36. Regla de Arbitraje CIADI. Regla 15(2).

37. M. Polkinghorne y C. B. Rosenberg, N. 2, pp. 126-127. 
de los árbitros ${ }^{38}$. Es evidente que el secretario arbitral no debe constituirse como el cuarto árbitro, decidiendo el caso y actuando de forma tal que implique una toma de decisiones sobre el fondo del asunto. El secretario debe limitarse a asistir, no a reemplazar el trabajo de los árbitros.

La Nota de la ICC 2016 numeral 84 adopta esta ideología, pues expresamente prohíbe al tribunal arbitral delegar la toma de decisiones. El tribunal arbitral no se puede apoyar en un secretario administrativo para realizar obligaciones esenciales que le corresponden al árbitro.

A su vez la Nota de la CNUDMI de 2016 en el párrafo 36 reconoce que los secretarios no se involucran y no participan en la toma de decisiones del tribunal arbitral, salvo en ciertos raros y especializados tipos de arbitraje, por ejemplo, cuando el reglamento de arbitraje dispone que se espera que los secretarios brinden consejería jurídica en relación a la decisión del tribunal arbitral y cuando el tribunal está compuesto de especialistas que no son abogados.

HKIAC Guidelines estipulan en el apartado 3.2 que el tribunal arbitral no debe delegar la toma de decisiones al secretario o apoyarse en el secretario para realizar cualquier obligación esencial del tribunal. JAMS Guidelines establecen que en ningún momento el secretario se puede comprometer a la toma de decisiones en nombre del tribunal arbitral.

\subsection{La remuneración del secretario administrativo}

Los altos honorarios del secretario han sido otra preocupación desde los años 90 en el seno de la ICC, misma que permanece vigente al día de hoy como se vio en el caso de Yukos. La facturación de Valasek estuvo cerca de ser igual a la de uno de los árbitros. Para evitar un incremento en los costos del arbitraje y tomando en cuenta que el secretario asistirá al tribunal en su 38. Ibídem. 
labor, resulta prudente que los honorarios del secretario sean deducidos de los honorarios de los árbitros y que los gastos así como viáticos que genere el Secretario sean cubiertos por las partes ${ }^{39}$. Este estándar adopta la Nota de la ICC de 2016 en sus numerales 89 a 91, los SCC Administrative Guidelines de 2014 y los numerales 4.3 y 4.5 de los HKIAC Guidelines.

La Nota de SIAC de 2015 contiene una solución diferente. Cuando la cantidad materia de la controversia sea de USD\$ $15^{\prime} 000,000^{40}$ o superior al momento de la solicitud de nombramiento del secretario, el tribunal arbitral puede acordar con las partes que los honorarios y gastos razonables del secretario sean pagados por las partes. Los honorarios del secretario están tazados a un máximo de USD $\$ 250$ por hora.

\section{Conclusiones}

Rusia -a diferencia de Sonatrach- sí logró anular los tres laudos parciales y tres finales que se dictaron en su contra ante el Juzgado de Distrito en La Haya, Países Bajos, pero no porque el asistente del tribunal Martin Valasek haya extralimitado sus funciones en la toma de decisiones. El Juzgado de Distrito en La Haya determinó que el tribunal arbitral no era competente para conocer del asunto y no entró al análisis de los otros puntos ${ }^{41}$. Esta es una de tantas otras batallas que Rusia libra ante las cortes de Alemania, Bélgica, Estados Unidos de América, Francia y Reino Unido donde Yukos intenta ejecutar los laudos dictados a su favor ${ }^{42}$.

A pesar de que el Juzgado de Distrito en La Haya no entró al estudio de las objeciones rusas en cuanto al papel de Martin Valasek, se puede decir que todas las funciones de orden adminis-

39. Ibídem.

40. Esta cantidad equivale aproximadamente a USD \$11'153,660.26, calculada a una tasa de cambio de un dólar de Singapur por USD \$0.74 al 24/09/2017.

41. Juzgado de Distrito de la Haya, The Russsian Federation v. Veteran Petroleum Limited, Sentencia de 20/04/2016, C/09/477160/HA ZA 15-1, párr. 5.97, 5.98, 6.1-6.9. <goo.gl/4zkBwm> (24/09/2017).

42. Véase, Shearman \& Sterling, <goo.gl/5y1hdw $>$. 
trativas resultan permisibles, tales como transmitir documentos y comunicaciones a las partes en nombre del tribunal arbitral; organizar y mantener el expediente; localizar documentos; organizar audiencias; presenciar audiencias, juntas y deliberaciones con el consentimiento de todos los miembros del tribunal; hacer notas y minutas; realizar investigaciones jurídicas y hasta una prueba de lectura para encontrar errores tipográficos, ortográficos y gramaticales o de cálculo en el laudo.

Incluso habría algunos argumentos a favor que PARTASIDES defendería para no otorgar la nulidad. Por ejemplo, PARTASIDES estaría a favor de que Valasek haya preparado un primer borrador de aquellas secciones del laudo donde (i) se identifiquen a las partes; (ii) la historia del procedimiento, siempre que la resolución de puntos procesales no haya sido controvertida; (iii) el señalamiento de los fundamentos concernientes a la competencia del tribunal arbitral siempre que no hayan sido controvertidos; $\mathrm{y}$, (iv) cuando los hechos de la controversia están suficientemente claros, se discutan los méritos del caso $^{43}$.

Empero, como ya vimos existen opiniones contrarias. Por una parte, DALHUISEN no comparte la idea que el secretario redacte cualquier decisión. Para él, los árbitros deben redactar la presentación de argumentos clave y la forma en que deben aparecer en el laudo final. Incluso, la redacción del secretario, aun y cuando sea bajo la dirección de los árbitros, le parece inapropiada. Este es el problema típico de diferentes expectativas que existe ante la ausencia de estándares claros que puedan balancear el procedimiento o decir qué es el debido proceso. Por eso es vital que existan instrumentos de soft law que permitan a las partes anticipar la toma de decisiones de un tribunal arbitral y los temas que puede delegar al secretario administratio.

Así, La Nota ICC de 2016 ilumina la zona gris. En este punto en particular conviene remitirse al párrafo 86 que refiere que el tribunal arbitral debe personalmente redactar cualquier decisión. La diferente forma de pensar que tienen PARTASIDES y DALHUISEN 43. C. Partasides, N. 14, p. 158. 
pueden ser motivos para elegirlos o no como árbitros, pero esto no necesariamente arroja un factor que brinde certidumbre a las partes a través de un resultado predecible en cuanto al actuar del secretario administrativo. Instrumentos de soft law son útiles para llegar a tal expectativa.

Los lineamientos de ICC y HKIAC resultan los más elaborados; consecuentemente, brindan mayor certidumbre a las partes, pues éstas pueden anticipar, hasta cierto punto, las funciones que el tribunal puede delegar al asistente, así como las obligaciones que implica desempeñar tal papel. Pero esto es un destello de luz. ¿Qué hay bajo los demás reglamentos de arbitraje? Una escala de grises donde no existe guía alguna para resolver algunos temas preocupantes. La escala de grises se agudiza en la medida que la discrecionalidad del tribunal arbitral es mayor y no consulta a las partes en la intervención del secretario.

Es cierto que un instrumento regulatorio limita la flexibilidad y discreción, pero el beneficio de una mayor regulación de soft law, puede mejorar la percepción de lo que es justo en la solución de controversias. Por esta y demás razones expuestas, es conveniente seguir desarrollando un marco similar al de la ICC y HKIAC que permita dar certidumbre a las partes del futuro a través de un marco predecible. 